\title{
Autocuidado en las personas mayores de la Ciudad de San Francisco de Campeche
} Self-care in older persons of San Francisco City of Campeche

\author{
Ana Rosa Can Valle \\ Universidad Autónoma de Campeche, México \\ anarocan@uacam.mx \\ Betty Sarabia Alcocer \\ Universidad Autónoma de Campeche, México \\ bmsarabi@uacam.mx \\ Jaqueline Guadalupe Guerrero Ceh \\ Universidad Autónoma de Campeche, México \\ jgguerre@uacam.mx
}

\section{Resumen}

En México las personas son consideradas adultas mayores a partir de los 60 años. La Organización Mundial de la Salud (OMS) menciona que el envejecimiento es el proceso fisiológico que inicia al nacer y que va sufriendo cambios durante todo el ciclo de vida. En la ciudad de San Francisco de Campeche residen 67879 adultos mayores, lo que representa $8.3 \%$ de la población total según el Instituto Nacional de Estadística y Geografía (INEGI. 2010).

El objetivo de este estudio es identificar si existe relación entre el autocuidado y las personas adultas mayores que asisten al Centro de rehabilitación y Educación Especial, en la Ciudad de San Francisco de Campeche. Para ello se utilizó el método descriptivo, exploratorio con alcance cualitativo, y los instrumentos Test de capacidad y percepción de Autocuidado del Adulto Mayor (CYPAC-AM). El análisis de datos se llevó a cabo con el programa SPSS. Los sujetos de estudio fueron 50 personas adultas mayores de más de 60 años. En los resultados se encontró que 32 sujetos de estudio son del sexo femenino y 18 del sexo masculino; del 100 \% de la población del género masculino, $44.44 \%$ muestra tener la percepción de autocuidado inadecuado; y del $100 \%$ 
de la población del género femenino, $68 \%$ presenta la percepción de parcialmente inadecuado. En la percepción del estado de salud, $60 \%$ considera tener una percepción de autocuidado de la salud parcialmente adecuada, $34 \%$ un nivel de autocuidado inadecuado, $3 \%$ sin capacidad de autocuidado, el nivel de autocuidado refleja $3 \%$ y el déficit total de autocuidado indica $0 \%$. En conclusión, en relación con el sexo y la percepción de autocuidado, la población total de hombres encuestados presentan un nivel de autocuidado adecuado, $11.11 \%$ menor al $50 \%$ estipulado, en comparación con el total de mujeres, quienes presentaron $0 \%$, lo que quiere decir que los hombres presentan mayor autocuidado que las mujeres.

Palabras clave: autocuidado, déficit de autocuidado, gerontología, vejez.

\section{Abstract}

In Mexico it is considered elderly a person from 60 years. According to WHO mentioned that aging is a physiological process that begins at birth and undergoes changes throughout the life cycle. In the city of San Francisco de Campeche reside 67879 aging, representing $8.3 \%$ of the total population according to the National Institute of Statistics and Geography (INEGI. 2010). Objective: to identify the correlation between self-care and the older persons attending the Center for Rehabilitation and Special Education in the City of San Francisco de Campeche. Method: a descriptive, exploratory qualitative scope. Instruments: test capacity and perception of self-care for the elderly (CYPAC-AM). Data analysis was performed using SPSS. Individuals: 50 adult seniors of 60 years. Results: we found that 32 respondents were female and 18 were male, that $100 \%$ of the male population sample $44.44 \%$ have inadequate self-perception and female $100 \%$ indicates $68 \%$ presents partially inadequate perception. In the perception of health status, $60 \%$ consider having a self-perception of the partially adequate health, $34 \%$ with an inadequate level of self-care, $3 \%$ showing no ability to self-care, self-care level that reflects the $3 \%$ and the total lack of self-indicating $0 \%$. Conclusion: about sex and perception of the self, the total population of male respondents have an adequate level of self-care $11.11 \%$ less than the $50 \%$ stipulated by comparing the total number of women had $0 \%$, this means that men have higher self than women.

Key words: self-care, self-care deficit, gerontology, aging. 
Fecha Recepción: Febrero 2015 Fecha Aceptación: Julio 2015

\section{Introducción}

La Organización de las Naciones Unidas considera anciana a toda persona adulta mayor de 65 años para los países desarrollados y mayor de 60 años para los países en desarrollo. Es decir, en México se considera adulta mayor a la persona a partir de que cumple los 60 años de edad. Según la OMS, el envejecimiento es un proceso fisiológico que inicia al nacer y que sufre cambios durante todo el ciclo de vida. En la ciudad de San Francisco de Campeche residen 67 879 adultos mayores, que representa al $8.3 \%$ de la población total según el Instituto Nacional de Estadística y Geografía (INEGI, 2010).

En los registros del Centro de Rehabilitación y Educación Especial (CREE) durante los meses de abril y mayo, ingresaron por primera vez 68 adultos mayores, mismos que se incluyen en esta investigación. Sus antecedentes científicos tiene cuatro vertientes: envejecimiento exitoso, salud y enfermedad en la persona mayor, funcionalidad en la vejez y autocuidado en la persona adulta mayor. La primera refiere un estado de bienestar; la segunda evoca la prevención, educación y promoción de la salud; la tercera trata sobre el nivel de dependencia y autonomía en actividades básicas, y la cuarta está enfocada en aquellas acciones que las personas mayores realizan para preservar su salud.

Al comienzo del nuevo siglo, las perspectivas de envejecimiento de las personas y de las poblaciones plantean algunos de los desafíos sociales, económicos y humanitarios más grandes que ha conocido la humanidad en su conjunto. En algún momento se pronosticó que entre los años 2011 y 2012 la población mundial llegaría a los 7000 millones de personas. Esto ya casi es un hecho; actualmente la gente vive más, lo que se documenta en los estudios demográficos, incluidos los de nuestro país, produciéndose una tendencia progresiva y exponencial al aumento cuantitativo, sobre todo en este sector de edad. Entre otras razones están los avances de la ciencia, particularmente la medicina, la cual ha disminuido o aplazado las causas que antaño 
provocaban la muerte, por ejemplo, enfermedades infecciosas, cardiovasculares y neoplásicas, siempre dentro del marco de desarrollo social.

Como consecuencia de este proceso de envejecimiento, en los países desarrollados la sociedad comienza a plantearse problemas sanitarios y sociales que hace poco tiempo eran escasamente considerados. Los factores socioeconómicos y psíquicos de estas personas también adquieren más protagonismo en la vejez, aumentando así la demanda de los servicios de salud (Balcázar G; Solórzano T., 2006).

Medir la salud en el paciente anciano es complejo debido a que se relaciona con medir la resultante de diversas variables de carácter biológico, psicológico y social, así que la salud del adulto mayor debe medirse en correspondencia con su funcionabilidad (Millán I., 2010).

Por tanto, el cuidado es una actividad permanente y cotidiana en la vida de los sujetos. Todos o casi todos se cuidan. Las demandas de cuidados aparecen por distintas circunstancias que se dan a lo largo de dicho proceso, o simplemente para promover la existencia de calidad. El autocuidado no es solo una función inherente a los profesionales de la salud, sino que también involucra al adulto mayor, a su familia, a sus vecinos a los grupos de voluntarios y a la comunidad en general, así que ellos tienen el potencial de convertirse en sus propios agentes de autocuidado e incorporar a su vida diaria conductas de salud que fomenten su bienestar integral para la solución de sus problemas. Ante lo descrito, esta investigación utiliza fundamentos basados en los argumentos de Orem (1995), sobre la Teoría de Déficit de Autocuidado enfocada en la persona adulta mayor.

\section{Autocuidado en la persona adulta mayor}

Actualmente es indiscutible el derecho de los adultos mayores a participar activamente en los programas de salud tanto en el sector público como privado, donde el autocuidado representa una alternativa eficaz para el logro de los objetivos, ya que al mejorar sus conocimientos sobre los aspectos de salud y control, la disminución de los factores de riesgo y la promoción de la conducta de autocuidado, se obtiene una población adulta mayor más saludable. Uno de los pilares filosóficos de la profesión de enfermería radica en el convencimiento del poder ilimitado 
que tiene el aprendizaje del ser humano, independientemente de su edad, posibilitando que a través del proceso educativo las personas de la tercera edad incorporen a su vida diaria conductas de salud física, social, mental y espiritual que favorezcan su bienestar, abandonando los estilos de vida que pueden perjudicarlas (OPS, 1993).

Larry C. Coppard et al. definen autocuidado como todas aquellas acciones y decisiones que toma un individuo para prevenir, diagnosticar y tratar su situación personal de enfermedad; todas las acciones individuales dirigidas a mantener y mejorar su salud; y las decisiones referidas a usar tanto los sistemas de apoyo informal como los servicios médicos formales. Es decir, el autocuidado es toda aquella acción individual que realiza el individuo por el bienestar de su persona, ya sea, físico, psicológico o social, con el fin de prevenir enfermedades o la complicación de las mismas (OPS, 1993).

El autocuidado significa aceptar la responsabilidad personal de la propia salud; mucha gente la practica sin asistencia de profesionales ya que solo se requiere de un poco de entrenamiento. El autocuidado significa adoptar estilos de vida saludable y hábitos de buena salud, tanto nutricionales como de ejercicio y relajación, con el apoyo de nuestra familia y amigos (González J., 2000).

El autocuidado es un concepto de vital importancia, ya que involucra a la persona mayor como actor competente capaz de tomar decisiones, controlar su propia vida y asegurarse la posibilidad de gozar de un buen estado de salud. En este sentido, el autocuidado permite alcanzar una mejor calidad de vida, a través del fortalecimiento del potencial de autonomía y de la responsabilidad de sí mismo, indispensables en el logro de un envejecimiento pleno y saludable (Araya A., 2012).

La mayoría de las personas mayores tienen la capacidad física y mental necesarias para realizar actividades de autocuidado a sí mismas o a otras personas. Sin embargo, esta posibilidad real es cuestionada por laicos, profesionales y los mismos adultos mayores, debido a conceptos equivocados y puntos de vista negativos sobre la vejez, al considerar que las personas de la tercera edad indiscutiblemente padecen de rigidez, enfermedad y discapacidad. Los individuos 
disponen de información sobre autocuidado a través de la escuela, los amigos, la familia, campañas de salud y vacunación, así como de los medios de comunicación (OPS, 1993).

Llevar a cabo el autocuidado a la salud es responsabilidad de cada individuo, así como preservarla de cualquier factor de riesgo que amenace su integridad. Por ello es importante conocer el nivel de preocupación que muestra la sociedad por mantener el equilibrio en salud. En la Asamblea Mundial de la Salud No. 42, celebrada en Ginebra en mayo de 1989, se estableció que la salud integral de los jóvenes debe ser considerada como un elemento básico para el desarrollo social y económico global. Disponer hoy en día de conocimiento sobre el autocuidado en salud debe ser un tema importante, en especial para la población joven, quienes son los que menos creen requerir supervisión médica o psicológica (Fuente V., 2011).

Actualmente los jóvenes adquieren dicha información por medio de las redes sociales y la tecnología, mientras que las personas mayores adquieren algunos de sus conocimientos básicos sobre autocuidado a través de sus experiencias cotidianas y tras observar situaciones que suelen afectar a la salud; sin embargo, es necesario que aprendan nuevos conocimientos y prácticas que les permitan realmente convertirse en sus propias fuentes de bienestar. Los adultos mayores, debido al envejecimiento más pronunciado en el que se encuentran, han aprendido a preocuparse y ocuparse de su salud, quedando bien definido este grupo etario a pesar de la suma de enfermedades. Algunos adultos mayores no solo son capaces de realizar actividades de autocuidado, sino que también pueden colaborar en el cuidado de otras personas con mayores limitaciones físicas y psicológicas gracias a sus experiencias de vida (OPS, 1993).

El autocuidado no solo es una función inherente a los profesionales de la salud, sino que también involucra al adulto mayor, a su familia, a los vecinos, a los grupos de voluntarios y a la comunidad en general. Este tipo de actividades es lo que se conoce como "cuidado laico" e implica que la persona comparta con otros la responsabilidad del cuidado de su salud. El individuo, solo o con ayuda de otros, se convierte de esa manera en su propio agente de cuidado. El autocuidado convierte a la persona de edad avanzada en motor de su propio bienestar y con el apoyo familiar y la participación de los recursos de la comunidad, puede encontrar la solución a la mayoría de sus problemas. La comunidad debe tomar parte activa en la promoción de la 
autosuficiencia, con el fin de reducir la situación de dependencia de los adultos mayores (OPS, 1993).

Nunca es tarde para iniciar medidas preventivas y cambios en los estilos de vida perjudiciales para la salud; por ejemplo, dejar de fumar, usar cinturones de seguridad, abstenerse de consumir alimentos que agraven las condiciones crónicas, mantener el tono y fuerza muscular mediante ejercicios, y realizar actividades que mejoren el funcionamiento orgánico y social, disminuyendo los efectos de las enfermedades que puedan surgir más adelante en la vida (OPS, 1993).

Diversos estudios han demostrado que las personas sedentarias de 60 años y más que deciden tomar parte en programas regulares de ejercicio mejoran su rendimiento cardiaco y disminuyen sus niveles de lípidos en la sangre. A menos que se logren mayores avances en la prevención y el tratamiento de las discapacidades vinculadas con la vejez, hará falta un mayor cupo en los hogares de ancianos y más camas en los hospitales para enfermedades graves, situación que vuelve imperativa la implementación de programas que promuevan la salud en estas personas, quienes requieren un elevado nivel de autocuidado (OPS, 1993).

\section{Metodología}

Esta investigación es de carácter descriptivo, y sus estudios buscan especificar las propiedades, las características y los perfiles de personas, grupos, comunidades, procesos, objetos o cualquier otro fenómeno que se someta a un análisis, por lo que se midió y recogió información de forma independiente sobre variables como sexo, edad, percepción del estado de salud, capacidad y percepción de autocuidado, así como el grado funcional por categorías. El diseño de la investigación es de tipo no experimental, es decir, no se variaron de manera intencionada las variables independientes para observar su efecto sobre otras variables (Hernández R y Fernández C., 2010). 


\section{Variables}

- Sexo: géneros masculino y femenino.

- Edad: la edad en años cumplidos dentro de tres intervalos: de 60 a 69 años, de 70 a 79 años, y de 80 años y más.

- Percepción del estado de salud: de acuerdo a la percepción del adulto mayor se clasificó en: aparentemente sano (saludable) y supuestamente enfermo (no saludable).

- Grado funcional: es el nivel de independencia del adulto mayor para realizar las actividades de la vida diaria, el cual se obtuvo de los resultados del primer ítem de cada una de las categorías funcionales evaluadas. Se clasificó en: dependiente e independiente.

- Capacidad y percepción de autocuidado: es el conocimiento y potencialidad del individuo para realizar actividades de autocuidado. Se clasificó en: percepción de autocuidado adecuado, percepción de autocuidado parcialmente adecuado, percepción de autocuidado inadecuado, sin capacidad de autocuidado y déficit de autocuidado total.

\section{Criterios de selección}

- Persona adulta mayor de 60 años o más de edad que asiste por primera vez al Módulo de Enfermería del Centro de Rehabilitación y Educación Especial, en el turno matutino en la Ciudad de San Francisco de Campeche.

- Persona adulta mayor de género indistinto.

- Adulto mayor consciente y orientado.

- Adulto mayor que desee participar en la investigación.

\section{Instrumento}

El Test de Capacidad y Percepción de Autocuidado del Adulto Mayor (CYPAC-AM), cuya autoría corresponde a Israel E. Millán Méndez 2010, es un cuestionario integrado por 8 categorías ordenadas de las letras A a la $\mathrm{H}$, las cuales fueron las siguientes: actividad física, alimentación, eliminación, descanso, sueño, higiene, confort, medicación, control de salud y hábitos tóxicos. Cada categoría está conformada por 4 ítems; el ítem 1 de cada categoría se forma por 2 incisos y los ítems 2, 3 y 4 por 3 incisos, con un valor máximo de 24 puntos y un valor mínimo de 0 . 
La Norma de Evaluación del Instrumento es el ítem 1 de cada categoría y determina el nivel de independencia o no para realizar esa actividad; si la respuesta es negativa, la persona es dependiente $\mathrm{y}$, por tanto, tiene déficit de autocuidado para esa categoría, si la respuesta es positiva, la persona es independiente para esa actividad y entonces se miden los otros ítems.

- Si hay tres o más respuestas positivas y ninguna respuesta negativa, se considera el autocuidado para esa categoría adecuado y se le da una puntuación de 3.

- Si hay dos o más respuestas intermedias (a veces), se considera el autocuidado para esa categoría parcialmente adecuado y se le da una puntuación de 2.

- Si hay más de una respuesta negativa, se considera el autocuidado para esa categoría inadecuado y se le da una puntuación de 1.

Rangos e Interpretación del Test Capacidad y Percepción de Autocuidado del Adulto Mayor (CYPAC-AM)

$\checkmark \quad$ Entre 21 y 24 puntos: percepción de autocuidado adecuado.

$\checkmark \quad$ Entre 16 y 20 puntos: percepción de autocuidado parcialmente adecuado.

$\checkmark \quad$ Con 15 puntos o menos, sin ninguna categoría evaluada de 0: percepción de autocuidado inadecuado.

$\checkmark \quad$ Con 15 puntos o menos, pero con al menos una categoría evaluada de 0: sin capacidad de autocuidado, o con déficit parcial para esa categoría.

$\checkmark \quad$ Con 0 en más de cuatro categorías evaluadas: con déficit de autocuidado total.

\section{Objetivo de la investigación}

Identificar si existe relación entre el autocuidado y las personas adultas mayores que asisten al Centro de Rehabilitación y Educación Especial de la Ciudad de San Francisco de Campeche.

\section{Resultados}

Esta investigación se realizó con personas adultas mayores que asisten al Centro de Rehabilitación y Educación Especial de la Ciudad de San Francisco de Campeche 


\section{Relación entre el sexo y la percepción de autocuidado}

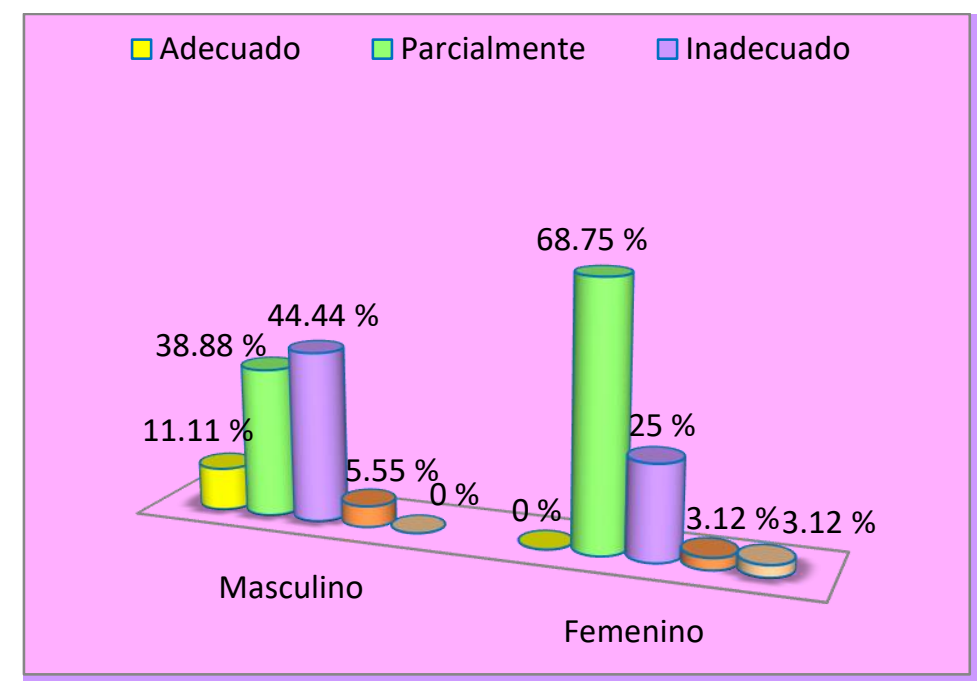

Figura 1. Relación entre el sexo y la percepción de autocuidado

En la figura 1 se muestra que del $100 \%$ de la población adulta mayor del género masculino, $44.44 \%$ tiene una percepción de autocuidado inadecuado, siendo este el resultado predominante, seguido de $38.88 \%$ con percepción de autocuidado parcialmente adecuado, $11.11 \%$ con percepción de autocuidado adecuado, $5.55 \%$ sin capacidad de autocuidado y $0 \%$ con déficit total de autocuidado.

En la misma gráfica se aprecia que del $100 \%$ de la población adulta mayor del género femenino, $68 \%$ indica una percepción de autocuidado parcialmente adecuado, siendo esta la cantidad más sobresaliente, $25 \%$ revela una percepción de autocuidado inadecuado, $3.12 \%$ sin capacidad de autocuidado, mientras que $3.12 \%$ arrojó déficit total de autocuidado y $0 \%$ manifestó tener una percepción de autocuidado adecuado.

En relación con el sexo y la percepción de autocuidado, los hombres predominaron con un nivel de autocuidado inadecuado de $44.44 \%$ y las mujeres con un nivel parcialmente adecuado de $68.75 \%$; por último, los hombres presentaron un nivel de percepción de autocuidado adecuado con $11.11 \%$ en comparación con las mujeres que mostraron $0 \%$. 


\section{Resultados de la relación entre la percepción de autocuidado y el rango de edad de las personas adultas mayores}

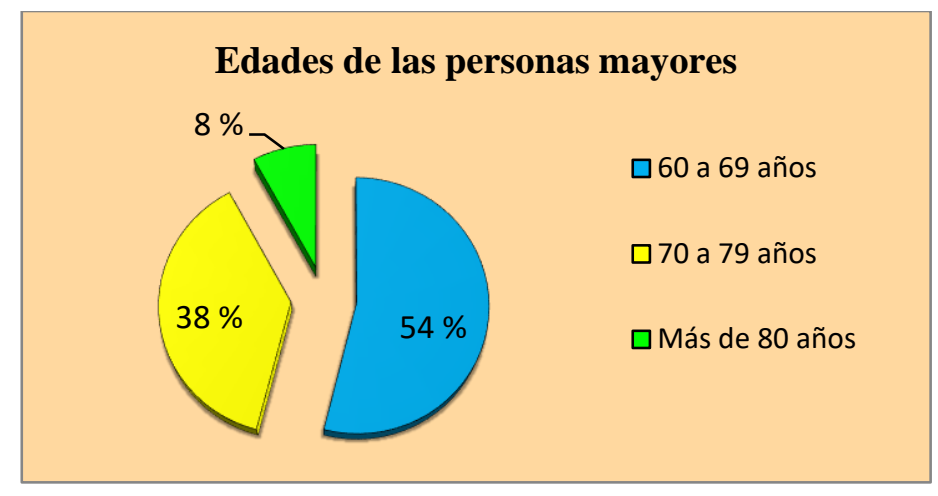

Figura 2. Personas adultas mayores de acuerdo a su edad

La figura 2 muestra el total de la población adulta mayor y su rango de edad, donde se puede apreciar que del $100 \%$ de las personas adultas mayores, $54 \%$ está dentro del rango de 60 a 69 años, $38 \%$ en el de 70 a 79 años y $8 \%$ en el de más de 80 años.

Percepción de autocuidado y su relación con la percepción del estado de salud de las personas adultas mayores que se consideran saludables y no saludables

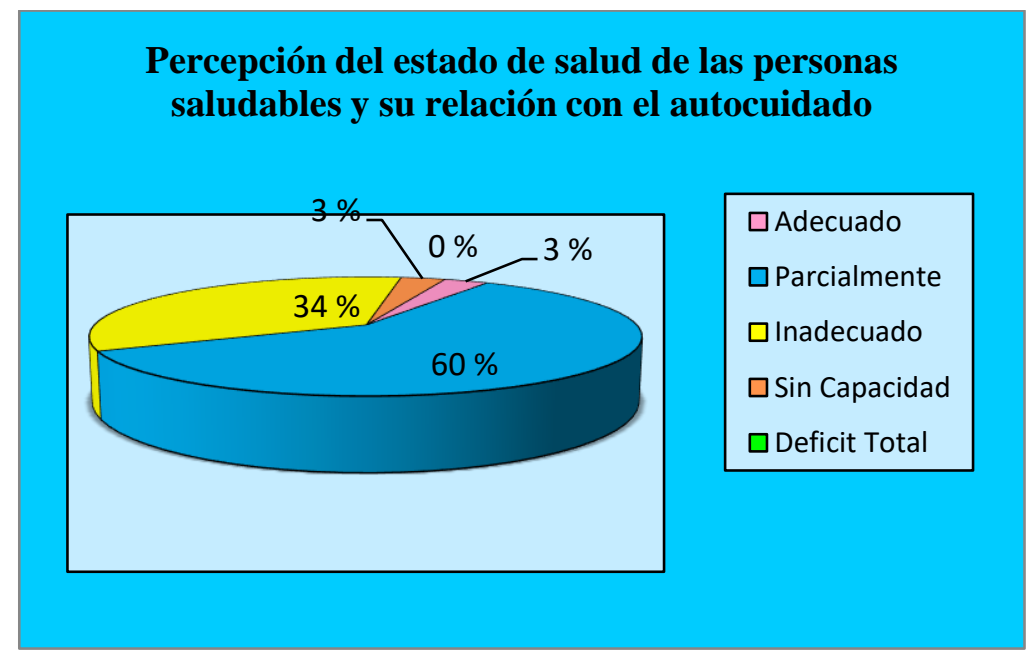

Figura 3. Percepción de estado de salud de las personas adultas mayores que se perciben saludables y su relación con la percepción de autocuidado. 
En la figura 3 se observa que del $100 \%$ de la población que se percibe saludable, $60 \%$ considera tener una percepción de autocuidado parcialmente adecuado, seguido del $34 \%$ con un nivel de autocuidado inadecuado, $3 \%$ que muestra sin capacidad de autocuidado, $3 \%$ con nivel de autocuidado adecuado y, por último, $0 \%$ con déficit total de autocuidado.

\section{Percepción del estado de salud de las personas adultas mayores que se perciben no saludables y su relación con la percepción de autocuidado}

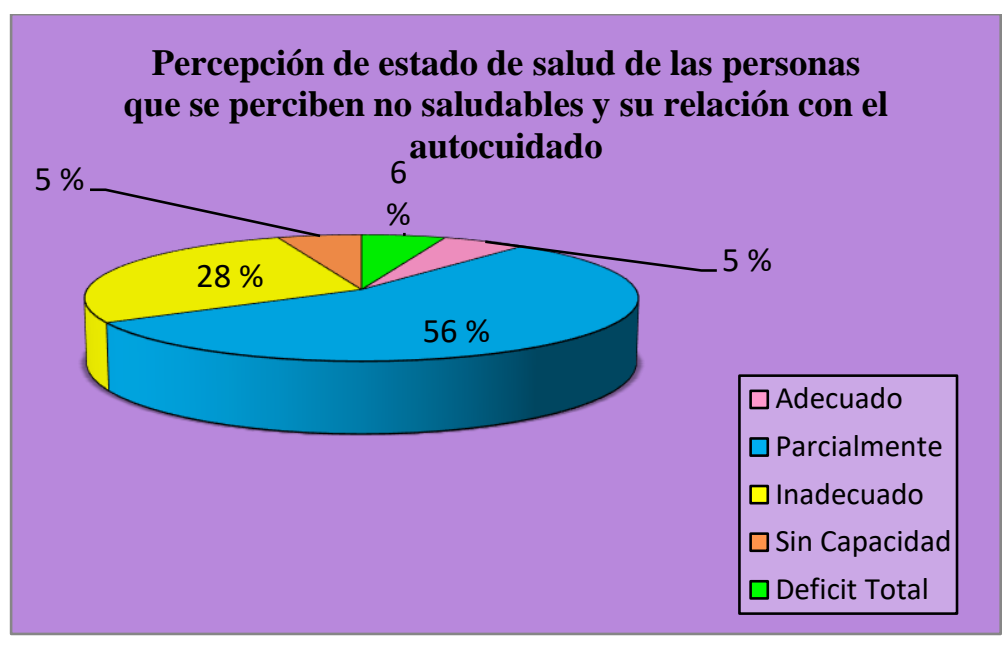

Figura 4. Percepción de estado de salud de las personas adultas mMayores que se perciben no Saludables y su relación con la percepción de autocuidado.

En la figura 4, la totalidad $(100 \%)$ de las personas adultas mayores que se perciben no saludables en relación con su percepción de autocuidado, 5 \% señala tener una percepción de autocuidado adecuado, $56 \%$ un autocuidado parcialmente adecuado, $28 \%$ reflejó un autocuidado inadecuado, $5 \%$ sin capacidad de autocuidado y $6 \%$ indica un déficit total de autocuidado. 


\section{Discusión}

Los resultados obtenidos muestran que en el rubro sexo y su relación con la percepción de autocuidado se considera que el género femenino tiene mayor autocuidado que los hombres por ser más dedicadas al bienestar de su familia y, por tanto, a sí mismas; aprenden de sus madres, y continúan con sus propias familias el denominado "cuidado maternal", por lo que se les considera un grupo responsable de mantener el estado de salud y bienestar de sus seres queridos. Sin embargo, el sexo masculino a pesar de ser considerado como el género fuerte, predomina como el grupo que menos cuida de su salud, debido a que se dedican más al ámbito laboral y el esfuerzo físico, así que el cuidado a la salud no se considera una actividad incorporada a su estilo de vida, por lo que no la perciben como una autorresponsabilidad necesaria para mantener su bienestar biopsicosocial. Sin embargo, para fundamentar estos resultados basta decir que otros autores mencionan que el sexo femenino es considerado como el sexo débil, con el estereotipo de ser cuidadoras de la salud de su familia pero no de su propia salud, es decir, cuando se trata de su autocuidado no lo realizan mejor o de manera más adecuada que sus cónyuges puesto que no lo consideran necesario en su proyecto de vida.

\section{Conclusión}

Con base en los resultados obtenidos, se concluye que de la población de estudio $36 \%$ equivale a hombres y $64 \%$ a mujeres. Así que hubo mayor cantidad de mujeres que de hombres encuestados. Podemos observar que del total de personas mayores de la ciudad de San Francisco de Campeche (67 879), los hombres predominaron con 34128 y las mujeres con 33 751, según los censos del INEGI en el año 2010, sin embargo, se presenta mayor asistencia de mujeres a áreas donde se brinda cuidado a la salud, como es el caso del Centro de Rehabilitación y Educación Especial (CREE), adonde asisten más mujeres que hombres.

A partir de esta hipótesis se demuestra que en relación con el sexo y la percepción de autocuidado, la población total de hombres encuestados presenta un nivel de autocuidado adecuado de $11.11 \%$, menor al $50 \%$ estipulado en comparación con el total de mujeres que presentaron $0 \%$, esto quiere decir que los hombres presentan mayor autocuidado que las 
mujeres, predominando en la población el autocuidado parcialmente adecuado en mujeres con $68.75 \%$ y el autocuidado inadecuado en hombres con $44.44 \%$.

En cuanto a la edad y la percepción de autocuidado, el total de las personas mayores de 60 a 69 años de edad muestran $74 \%$ de autocuidado parcialmente adecuado, los de 70 a 79 años muestran $48 \%$ de autocuidado inadecuado y, por último, las personas de más de 80 años muestran $25 \%$ en autocuidado adecuado, parcialmente adecuado, sin capacidad de autocuidado, y déficit total de autocuidado, lo cual quiere decir que las personas adultas mayores a mayor edad presentan una disminución de autocuidado, donde la población mayor de 80 años demuestra una mínima cantidad de autocuidado adecuado, menor al $50 \%$.

Con relación a la percepción del estado de salud y la percepción de autocuidado, observamos que las personas mayores que se consideran saludables, $60 \%$ presenta un autocuidado parcialmente adecuado, esto significa que menos de la mitad de los que se sienten relativamente sanos, tienen un nivel de autocuidado adecuado; y los que se consideran aparentemente enfermos, poseen 56 $\%$ de autocuidado parcialmente adecuado. Esto quiere decir que de la población total que se considera saludable y no saludable, menos de la mitad se encuentra con autocuidado adecuado.

De la población total de personas adultas mayores independientes en las 8 categorías de medición, $4 \%$ muestra un autocuidado adecuado, esto quiere decir que menos del $10 \%$ de las personas independientes pueden elegir autocuidarse correctamente o no, lo que demuestra que son independientes pero no se cuidan correctamente. En la población de personas con al menos 1 categoría evaluada de 0 considerada dependiente, presentan un nivel de autocuidado adecuado de $0 \%$, esto expresa que la funcionalidad del paciente influye en la capacidad de autocuidado, por lo que a mayor dependencia menor autocuidado. 


\section{Recomendaciones}

\section{Persona adulta mayor}

-Integrarse a grupos o talleres para compartir experiencias, ofrecer puntos de vista en las redes sociales, mejorar la autoestima e integración familiar con el fin de fortalecer el autocuidado psicosocial.

-Fomentar la elección de conductas saludables y adoptar hábitos de buena salud, tanto nutricionales como de ejercicio físico y mentales, mejorando así la calidad de vida.

-Promover el cuidado de sí mismos, mediante la promoción a la salud y prevención de enfermedades e incapacidades, asistiendo a talleres, clases o capacitaciones sobre autocuidado en el adulto mayor para lograr un estilo de vida saludable.

-La persona adulta mayor debe asumir la responsabilidad del cuidado de su propia salud.

\section{Familiar o cuidador primario}

-Informar al familiar o cuidador primario de los cursos y talleres de capacitación que imparte el profesional de salud especializado en la atención integral del adulto mayor.

-Asistir a programas de respiro, que consisten en ofrecer temporalmente alojamiento a personas dependientes de forma que sus cuidadores puedan liberarse de la carga de los cuidados durante el tiempo que dura la estancia.

-Contar con una mayor flexibilidad laboral para hacer más agradable la atención de cuidar, fomentando una mejor relación entre el cuidador primario y la persona adulta mayor.

-Conocer la enfermedad o impedimento de la persona dependiente, su posible evolución y complicaciones, haciendo que el cuidado sea más efectivo.

\section{Personal de salud}

-Capacitar al personal de salud sobre envejecimiento para brindar una mejor atención y promoción de la salud a las personas adultas mayores sobre su cuidado.

-Realizar una mejor promoción de la salud y prevención de enfermedades mediante el conocimiento adecuado de las necesidades que envuelven a la persona adulta mayor en las esferas biopsicosociales y lograr así una atención integral y la satisfacción del cliente. 
-Concientizar al personal de salud en su cuidado, propiciando el interés de autocuidarse, mediante la asistencia a talleres, conferencias y cursos sobre su autocuidado, con el fin de lograr un envejecimiento saludable y exitoso.

\section{Licenciados en gerontología}

-Retomar esta investigación realizando el estudio en otros grupos de personas adultas mayores y estratos socioeconómicos, con el fin de contribuir a la investigación.

-Elaborar manuales de autocuidado en el adulto mayor, que aporten información valiosa a las instituciones de salud y mejoren la atención a este grupo de la población.

-Aportar conocimientos sobre la atención integral de las personas mayores, realizando talleres, conferencias, capacitaciones y cursos al personal de las instituciones de salud y población en general, para mejorar la calidad de vida de la población mayor.

\section{Bibliografía}

Bazo, M. (2005). Envejecimiento y sociedad: una perspectiva internacional. Buenos Aires: Médica Panamericana.

Díaz, F; Alemán, M., y Jaimes, E. (2004). Medicina preventiva. En M. Alemán (Ed.), Tópicos de Geriatría (pp. 75-78). México: Prado.

Fernández, R. (2000). Gerontología social. Una introducción. En R. Fernández. (Ed.), Gerontología social. Madrid, España: Pirámide.

González, J. (2000). Manual de autocuidado y salud en el envejecimiento. $6^{\mathrm{a}}$ ed., México: CostaAmic.

Hernández, R; Fernández, C; Baptista, P. (2010). Metodología de la Investigación. 5 ${ }^{\text {a ed., }}$ México: McGraw-Hill.

Organización Panamericana de la Salud (1993). Enfermería Gerontológica: conceptos para la práctica: Oficina Sanitaria Panamericana. 
Marin, P; Gac, H; Carrasco, M. (2007). Geriatría y Gerontología. Buenos Aires: Universidad Católica de Chile.

Pérez, G. (2004). Prevención de la salud con personas mayores. En M. Fernández (Ed.), ¿Cómo intervenir en personas mayores? 2a . ed., Madrid, España: Dykinson.

Pinazo, S; Sánchez, M. (2005). Gerontología. Actualización, innovación y propuestas. Madrid: Pearson Educación.

Rodríguez, R., y Lazcano, G. (2007). Envejecimiento exitoso. En R. Rodríguez (Ed.), Práctica de la Geriatría. México: McGraw-Hill.

“Guía Médica y de la salud para mayores de cincuenta años” (2000). México: Trillas.

Barrero, C; García, S; Ojeda, A. (2005). Índice de Barthel (IB): Un instrumento esencial para la evaluación funcional y la rehabilitación. En Plasticidad y Restauración Neurológica, (4), 81-85. Recuperado dehttp://www.medigraphic.com/pdfs/plasticidad/prn2005/prn051_21.pdf

Buil, P; Díez J. (2004). Anciano y familia. Una relación en evolución. En Anales, 22 (1) 19-25. Recuperado de www.cfnavarra.es/salud /anales /textos/vol22/suple1/suple3.html

Brigeiro, M. (2005). Envejecimiento exitoso y tercera edad: Problemas y retos para promoción de la salud. En Investigación y Educación en Enfermería, 23(1), 102-109. Recuperado de http://www.redalyc.org/ articulo.oa?id=105215401009\#

Castillo, M. (2009). Envejecimiento exitoso. En Medica Clínica Condes, 20 (2), 167-174. Recuperado de www.clc. cl/Dev_CLC/media/ Imagenes/PDF\%20revista \%20m\%C 3\%A9dica /2009/2\%20marzo/ 04ENVEJECIMIENTO-4.pdf

Fuente, V. (2011). Conocimiento y acciones de los adolescentes sobre el autocuidado de su salud. En Conamed, 16 (1), 29-33. Recuperado de http://dialnet.unirioja.es/servlet/articulo?Codi go=3640038.

González, Sara; Moreno, Pérez (2011). Instrumentos para la enseñanza del proceso de enfermería en la práctica clínica docente con enfoque de autocuidado docente con enfoque de autocuidado utilizando Nanda-Nic-Noc. En Enfermería Global, 10 (23), 89-95. Recuperado de http://scielo.isciii.es/pdf/eg/v10n23/docencia1.pdf 
Gorete, R; Teixeira, L; Paúl, C; Martins, M. (2012). Independencia en las actividades de la vida. Estudio de validación de una escala para la población portuguesa. En Gerokomos, 23 (2), 69-73. Recuperado de http://scielo.isciii.es/pdf/geroko/v23n2/comunicacion 4.pdf

Millán, Israel (2010). Evaluación de la capacidad y percepción de autocuidado del adulto mayor en la comunidad. En Revista cubana de Enfermería, 26 (4), 222-234. Recuperado de http://scielo.sld. cu/pdf/enf/v26n4/en f07410.pdf

Navarro, P; Castro, S. (2001). Modelo de Dorothea Orem aplicado a un grupo comunitario a través del proceso de enfermería. En Enfermería Global, 19, 1-14. Recuperado de http://scielo.isciii.es/pdf/eg/n19/clinica3.pdf

Rivero, Un; et al. (2014). Valoración ética del modelo de Dorothea Orem. En Ciencias médicas, 6 (39, 0-0. Recuperado de http://scielo.sld.cu/pdf/rhc m /v6n3/r hem12307.pdf

Sarabia, C. (2009). Envejecimiento exitoso y calidad de vida. Su papel en las teorías del envejecimiento. En Gerokomos, 20 (4), 172-174. Recuperado de http://scielo.isciii.es/scielo.php?script=sci_arttext\&pi d=S1134-928X2009 000400005

Sanhueza, M; Castro, M; Merino, J. (2005). Adultos mayores funcionales: nuevo concepto en salud. En Gerokomos, (2), 17-21. Recuperado de http://www.scielo.cl/scielo.php?script=sci_arttext \&pid=S0717-9553200500 0200004

Segovia, M; Torres, E. (2011). Funcionalidad del adulto mayor y el cuidado enfermero. En Gerokomos, 22(4), 162-166. Recuperado de http://scielo.isciii.es/pdf/geroko/v22n4/comunica ción2. pdf

Trigás, M; Ferreira, L; Meijide, H. (2011). Escalas de valoración funcional en el anciano. En Galicia Clínica, 72 (1), 11-16. Recuperado de http://www.galiciaclinica.info/PDF/11/225.pdf

Acosta, C; Dávila, M; Rivera, M., y Rivas, J. Actividades de la vida diaria y envejecimiento exitoso. Recuperado de http://www.geriatria.salud.gob.mx/ descargas /15.pdf

Aguirre, H; Ruiz, L. (2008). Seminario el ejercicio actual de la medicina. Envejecimiento exitoso. Recuperado de http://www.facmed.unam.mx/sms/seam2K1/2008/sep_01_ponencia.htm 
Araya, A. (2012). Cartillas educativas calidad de vida en la vejez. Autocuidado de la Salud. Recuperado de http://adultomayor.uc.cl/docs/autocuidado_salud.pdf.

Francke, L; González, B; Lozano, L. (2011). Envejecimiento exitoso, una tarea de responsabilidad individual. En Ama y trasciende. Recuperado de http://amaytrasciende.org/docs/publicos/

Envejecimiento\%20\%C2\%A0exitoso,\%20\%C2\%A0una\%20\%C2\%A0tarea\%20\%C2\%A0 de $\% 20 \% \mathrm{C} 2 \%$ A0responsabilidad $\% 20 \% \mathrm{C} 2 \%$ A0individual.pdf.

Leitón, Z; Ordóñez, Y. (2003). Autocuidado de la salud para el adulto mayor. Manual de información para profesionales. Recuperado de http://es.slideshare.net/sonita1801/manualde-informacion-para-profesionales-autocuidado-de-la-salud-para-el-adulto-mayor.

Meneu, R. (2001). Interculturalidad y extensión de la Cobertura de la Protección Social en Salud para trabajadores agrícolas y pueblos indígenas. Recuperado de http://biblioteca.programaeurosocial.eu/ PDF/Salud/Modulo3.pdf.

Rodríguez, K. (2010). Vejez y envejecimiento. Recuperado de http://www.urosario.edu.co/urosario_files/dd/dd857fc5-5a01-4355-b07ae2f0720b216b.pdf.

Rusbel, E. (2013). La eutanasia en pacientes en fase terminal: una visión desde la licenciatura de educación para la salud. Recuperado de http://sdyms2014a.blogspot.mx/ 